\title{
Next-to-Leading-Order Predictions for $\boldsymbol{t}$-Channel Single-Top Production at Hadron Colliders
}

\author{
J. M. Campbell, ${ }^{1}$ R. Frederix, ${ }^{2,3}$ F. Maltoni, ${ }^{3}$ and F. Tramontano ${ }^{4}$ \\ ${ }^{1}$ Department of Physics and Astronomy, University of Glasgow, Glasgow G12 8QQ, United Kingdom \\ ${ }^{2}$ PH Department, Theory group, CERN 1211-CH Geneva, Switzerland \\ ${ }^{3}$ Center for Particle Physics and Phenomenology (CP3), Université catholique de Louvain, B-1348 Louvain-la-Neuve, Belgium \\ ${ }^{4}$ Università di Napoli Federico II, Dipartimento di Scienze Fisiche, and INFN, Sezione di Napoli, I-80126 Napoli, Italy
}

(Received 3 March 2009; published 8 May 2009)

\begin{abstract}
We present the predictions at next-to-leading order (NLO) in the strong coupling for the single-top cross section in the $t$ channel at the Tevatron and the LHC. Our calculation starts from the $2 \rightarrow 3$ Born amplitude $g q \rightarrow t \bar{b} q^{\prime}$, keeping the $b$-quark mass nonzero. A comparison is performed with a traditional NLO calculation of this channel based on the $2 \rightarrow 2$ Born process with a bottom quark in the initial state. In particular, the effect of using kinematic approximations and resumming logarithms of the form $\log \left(Q^{2} / m_{b}^{2}\right)$ in the $2 \rightarrow 2$ process is assessed. Our results show that the $2 \rightarrow 3$ calculation is very well behaved and in substantial agreement with the predictions based on the $2 \rightarrow 2$ process.
\end{abstract}

DOI: 10.1103/PhysRevLett.102.182003

PACS numbers: $12.38 . \mathrm{Bx}, 14.65 . \mathrm{Ha}$

It is a quite remarkable fact that in hadron collisions, top quarks can be produced via electroweak interactions at a rate comparable with strong production [1-3]. Such unique behavior is mainly due to two factors. First, a top quark can be produced together with its $S U(2)_{L}$ partner, the bottom quark, with a sizable gain in phase space cost with respect to a top and antitop quark pair. Second, among the three possible production channels, one entails the exchange of a vector boson in the $t$ channel, leading to an enhanced cross section at high energies.

Given the large predicted cross section, evidence for single-top production has been actively sought and recently established at the Tevatron [4,5] and it will play an important role in the physics program at the LHC. Single-top production offers, for instance, the only effective way of extracting direct information on $V_{t b}$ [6]. In fact, at the Tevatron the prospects for the detection and then measurement of the electroweak (EW) production cross sections have significantly worsened since the first theoretical proposals [7]. The main reason for this was an underestimate of the impact of large backgrounds such as those coming from $W+$ jet production (both with and without heavy flavors) and from the strong production of $t \bar{t}$ [8]. The situation at the LHC, though bound to improve thanks to the larger rates expected, will not be qualitatively very different.

The most accurate analyses for single top are based on two essential ingredients. The first is an in situ determination of the background rates. Predictions from theory are in this case not able to match the needed accuracy. The second is the systematic exploitation of theoretical predictions for the kinematic properties of signal (and backgrounds). This information is encoded via sophisticated analysis techniques (such as those based on matrix elements, neural networks, and others [4,5]). Such methods are crucial in building efficient discriminating variables to select the standard model signal or possibly find indications of new physics effects [9].

It is therefore clear that the most accurate predictions for the signal, both for rates and kinematic distributions, are needed as inputs in these analyses. An intense activity in the last 15 years has led to increasingly sophisticated predictions at next-to-leading order (NLO) accuracy. Calculations have progressed from evaluations of total rates [10,11], to differential distributions $[12,13]$, including spin correlations in production and decay [14-17] and finally to the implementation of the three production channels in a fully exclusive Monte Carlo program $[18,19]$.

All NLO calculations available so far are based on the $2 \rightarrow 2$ scattering process, Fig. 1(a), where a $b$ quark appears in the initial state $[20,21]$. The usefulness of such an approach, called the five-flavor (5F) scheme, is twofold. First, the calculation greatly simplifies (as we shall describe in detail later), leading to straightforward calculations and compact results. Second, possibly large logarithms of the form $\log \left(Q^{2} / m_{b}^{2}\right)$ due to initial state collinear configurations with $g \rightarrow b \bar{b}$ splitting are consistently resummed into the $b$-quark parton distribution functions leading to an improved stability of the perturbative expansion. Effects related to the "spectator $b$," such as the presence of a $b$ jet and the $b$ mass, Fig. 1(b), only enter at NLO. As a result, most of the current calculations and corresponding Monte Carlo implementations [12,14-19] do not accurately model such effects. An alternative ap-

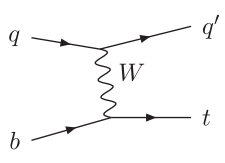

(a)

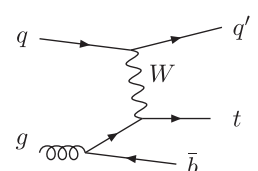

,

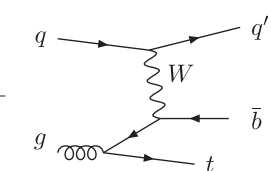

(b)
FIG. 1. Diagrams contributing at LO in the $2 \rightarrow 2$ (a) and $2 \rightarrow$ 3 (b) approaches. 
proach is to consider as Born the $2 \rightarrow 3$ scattering process, Fig. 1(b), keeping a finite $b$ mass. In this scheme, called the four-flavor (4F) scheme, the $b$ quarks do not enter in the QCD evolution of the PDF's and of the strong coupling. The calculation of the NLO corrections is much more involved due to the inclusion of an additional parton in the final state and the presence of a further mass scale. However, features associated with the kinematic description of the spectator $b$ 's can be genuinely investigated at NLO accuracy. In this Letter we present NLO results in the $4 \mathrm{~F}$ scheme and compare them to those in the $5 \mathrm{~F}$ scheme. The two approaches, being by definition equivalent, would give the same results at all orders in the perturbative expansion. At fixed (low) order, however, predictions could in principle differ significantly and the question of the range of applicability of each approach is raised.

A very important simplification in the $2 \rightarrow 2$ calculation at NLO is that QCD corrections completely factorize in terms of light and heavy currents: color conservation forbids the interference between diagrams (one-loop-Born or real-real) where the light quark and the heavy quark lines are connected by a gluon. In addition, real-real interferences between $t$-channel [Fig. 1(b)] and $s$-channel [Fig. 2(a)] diagrams vanish. A clear separation between the two processes is therefore maintained at NLO.

Quite remarkably, QCD corrections to the $2 \rightarrow 3$ Born still display almost a complete factorization. Most of the real emission processes can be uniquely associated (i.e., in a gauge invariant way) with either the light quark current or the heavy quark current. Interference terms are either exactly zero or color suppressed by $1 / N_{c}^{2}$. We have kept the color-suppressed interferences between light and heavy currents, but not those between $s$-channel NNLO real corrections and the $t$-channel real corrections [depicted in Fig. 2(b)]. Interferences in $q \bar{q} \rightarrow t \bar{b} q^{\prime} \bar{q}$ subprocess between $t$-channel diagrams and those with an on-shell $W$, $q \bar{q} \rightarrow t \bar{b}\left(W^{-} \rightarrow q \bar{q}^{\prime}\right)$ vanish in the $W$ zero-width limit and have not been included. However, we have checked that all the neglected interferences are very small and de facto do not hamper a meaningful separation of the channels.

All of the analytic calculations presented in this Letter have been performed with the FORM program [22]: treelevel and loop matrix elements are computed at the helicity amplitude level and therefore top spin information is avail-

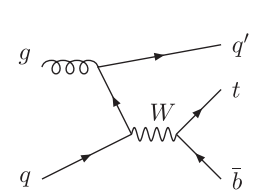

(a)

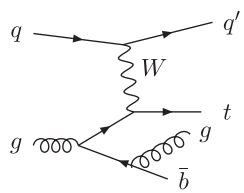

(b)

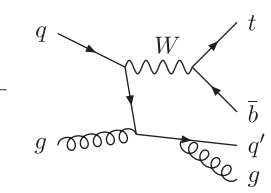

b)
FIG. 2. (a) Representative $s$-channel diagram present in $g q \rightarrow$ $t \bar{b} q^{\prime}$. Interference of these diagrams with those of Fig. 1(b) vanishes because of color. (b) Representative $t$ - and $s$-channel diagrams for $g q \rightarrow t \bar{b} g q^{\prime}:$ their interference is suppressed by $1 / N_{c}^{2}$. able. Tree-level matrix elements and the LO results for cross sections and distributions have been accurately checked with MadGraph/MadEvent Monte Carlo event generator [23]. The loop contributions have been evaluated in both the dimensional reduction and four-dimensional helicity schemes, following the procedure outlined in Ref. [12]. Tensor integrals have been decomposed with the help of a reduction routine based on the PassarinoVeltman approach [24]. Scalar integrals have been explicitly computed with standard methods and compared numerically with those of Ref. [25]. Gauge invariance, $C P$, and kinematic symmetries $\left(m_{b} \leftrightarrow m_{t}\right)$ have been extensively used to check the consistency of the calculation. Infrared and collinear divergences in the integrated real and virtual contributions have been canceled locally through the use of the dipole subtraction technique [26] in its massive formulation [27] as implemented in the Monte Carlo MCFM program [28]. The contributions from the dipole counterterms have been generated independently by the MadDipole code [29] and checked pointby-point in phase space. Finally, as the most important check of our calculation, we have derived the results for $e^{+} e^{-} \rightarrow Z / \gamma^{\star} \rightarrow b \bar{b} g$ at NLO. This calculation can be obtained from ours by simply accounting for the difference in the weak couplings, by setting $m_{b}=m_{t}$, by ignoring the QCD corrections on the light current and by setting the $W$ virtuality positive. We found excellent agreement with the results of Ref. [30], that have been obtained in completely different subtraction and regularization schemes.

We now present and discuss the results of the NLO calculation in the $4 \mathrm{~F}$ scheme (Born $2 \rightarrow 3$ ) and compare with those of the $5 \mathrm{~F}$ scheme (Born $2 \rightarrow 2$ ), at the Tevatron $(p \bar{p}, \sqrt{s}=1.96 \mathrm{TeV})$ and the LHC $(p p, \sqrt{s}=14 \mathrm{TeV})$. In our studies we assume $m_{t}=172 \mathrm{GeV}, m_{b}=4.7 \mathrm{GeV}$ and use the CTEQ6.6 PDF set [31]. For the $2 \rightarrow 3$ calculation we pass to the $4 \mathrm{~F}$ scheme by adding suitable finite terms, as explained in Ref. [32]. As an independent check we have verified that results obtained with the explicit fourflavor MRST set [33] are fully consistent with those obtained in the corresponding five-flavor MRST set plus the finite terms. In order to perform a fair comparison between the two schemes, we strictly follow the approach of Refs. [20,21] and, contrary to most of the available MC implementations [12,14-19] in the 5F scheme, we compute the real diagrams $g q \rightarrow t \bar{b} q^{\prime}$ with a nonzero $b$ mass. We have checked that this has a negligible effect on the total cross sections, as well as in the top and light jet distributions at the Tevatron [34] and the LHC. On the other hand, the distributions of the spectator $b$ 's are significantly affected.

In Fig. 3 we show the cross sections for top production at the Tevatron and the LHC in the two schemes as a function of $\mu / m_{t}$, where $\mu$ is a common renormalization and factorization scale. The $4 \mathrm{~F}$ calculation has a stronger dependence on the scale than the $5 \mathrm{~F}$ one, particularly at the Tevatron, which simply reflects the fact that the $2 \rightarrow 3$ Born calculation already contains a factor of $\alpha_{s}$. 


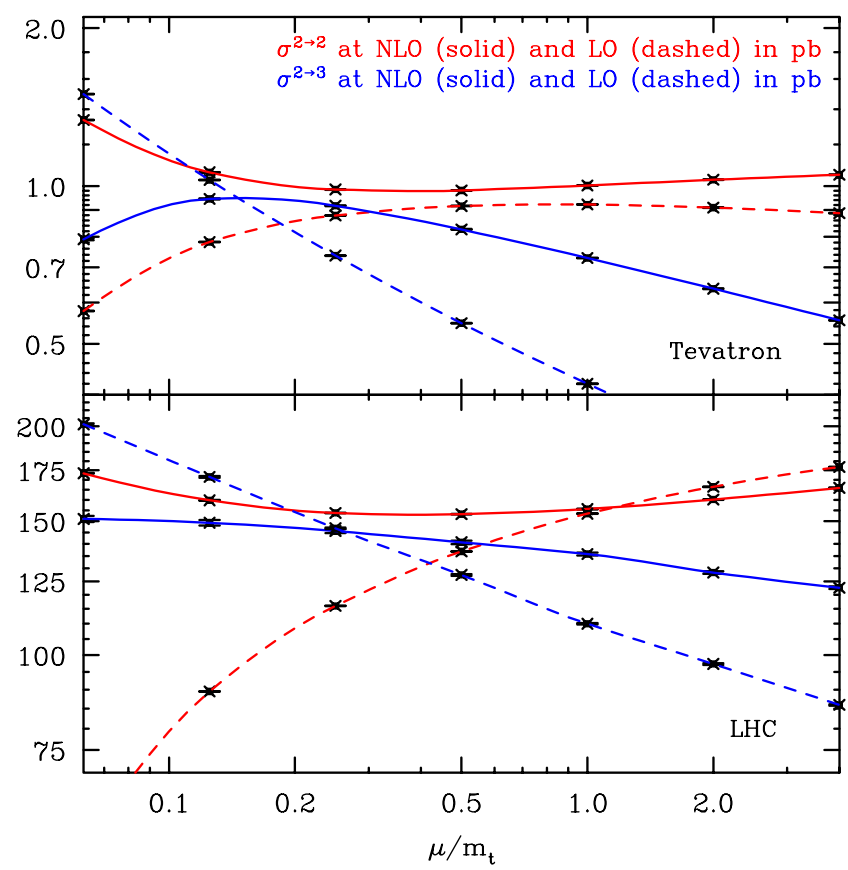

FIG. 3 (color online). Scale dependence of the $2 \rightarrow 2$ and $2 \rightarrow$ 3 calculations, at LO (dashed) and NLO (solid) order. Factorization and renormalization scales in the heavy and light quark lines are equal to $\mu$. For the LHC only top production is considered, the behavior of the antitop being very similar.

However, we observe that both calculations are much more stable under scale variations at NLO than at LO. To establish an optimal central value for the scales, we have studied separately the scale dependence associated with the light and heavy quark lines. As expected, most of the overall scale dependence is inherited from the heavy quark line. In the $4 \mathrm{~F}$ scheme it is minimal for scales around $m_{t} / 2$ and $m_{t} / 4$ for the light and heavy quark lines, respectively, which therefore sets our central scale choice. In the $5 \mathrm{~F}$ scheme the scale dependence is very mild and we simply choose $m_{t}$ for both lines.

Table I shows the predictions for the total cross sections in the two schemes, together with their uncertainties. The scale uncertainties are evaluated by varying the renormalization and factorization scales independently between $\mu_{0}^{L, H} / 2<\mu_{F, R}<2 \mu_{0}^{L, H}$ with $1 / 2<\mu_{F} / \mu_{R}<2$ and

TABLE I. Inclusive cross sections (in pb) for $t$-channel singletop production at the Tevatron and LHC using (CTEQ6L1) CTEQ6.6 PDF's for the (LO) NLO predictions and $\mu_{0}^{L}=m_{t}$ $\left(\mu_{0}^{H}=m_{t}\right)$ and $\mu_{0}^{L}=m_{t} / 2\left(\mu_{0}^{H}=m_{t} / 4\right)$ as central values for the factorization and renormalization scales for the light (heavy) line in the $5 \mathrm{~F}$ and $4 \mathrm{~F}$ schemes, respectively. The first uncertainty comes from scale variations, the second from PDF errors.

\begin{tabular}{lccc}
\hline \hline Born & $\begin{array}{c}\text { TeV } t(=\bar{t}) \\
(\mathrm{LO}) \mathrm{NLO}\end{array}$ & $\begin{array}{c}\text { LHC } t \\
(\mathrm{LO}) \mathrm{NLO}\end{array}$ & $\begin{array}{c}\text { LHC } \bar{t} \\
\text { (LO) NLO }\end{array}$ \\
\hline $2 \rightarrow 2$ & $(0.92) 1.00_{-0.02-0.08}^{+0.03+0.10}$ & $(153) 156_{-4-4}^{+4+3}$ & (89) $93_{-2-2}^{+3+2}$ \\
$2 \rightarrow 3$ & $(0.68) 0.94_{-0.11-0.07}^{+0.07+0.08}$ & (143) $146_{-7-3}^{+4+3}$ & (81) $86_{-3-2}^{+4+2}$ \\
\hline \hline
\end{tabular}

$\mu^{L} / \mu^{H}$ constant. We see that the uncertainty in the $4 \mathrm{~F}$ scheme is larger than (similar to) that in the $5 \mathrm{~F}$ scheme at the Tevatron (LHC). The difference between the NLO predictions in the two schemes is rather small, with uncertainties typically less than $5 \%$ in both cases. The exception is the $4 \mathrm{~F}$ calculation at the Tevatron with an uncertainty of around $10 \%$, which is, however, still of the same order as the absolute difference with the $5 \mathrm{~F}$ calculation. The small scale uncertainties together with quite modest increases of the cross sections from LO to NLO provide a clear indication that the perturbative expansions are very well behaved.

In Fig. 4 we compare NLO predictions for the top quark and light jet pseudorapidity $\eta$ and transverse momentum $p_{T}$. To define the light jet we used the $k_{T}$ algorithm and imposed $p_{T}>15 \mathrm{GeV}, \Delta R>0.7$. Results are presented as a bin-by-bin ratio of the normalized (4F and $5 \mathrm{~F}$ ) distributions. For the LHC only top production is shown, with the behavior of the antitop very similar. Although the predictions differ somewhat, the differences are typically at the $10 \%$ level and always less than $20 \%$. Finally, we study the NLO distributions in $\eta$ and $p_{T}$ for the spectator $b$. We find that the fraction of events at the Tevatron (LHC) where the $b$ is central and at high- $p_{T}\left(|\eta|<2.5, p_{T}>\right.$ $20 \mathrm{GeV}$ ) is $28 \%(36 \%)$ with a very small scale dependence. From Fig. 5 we see that the largest effects in the shapes are present at the Tevatron, where the spectator $b$ tends to be more forward and softer at high $p_{T}$ than in the $5 \mathrm{~F}$ calculation (where these observables are effectively only at LO).
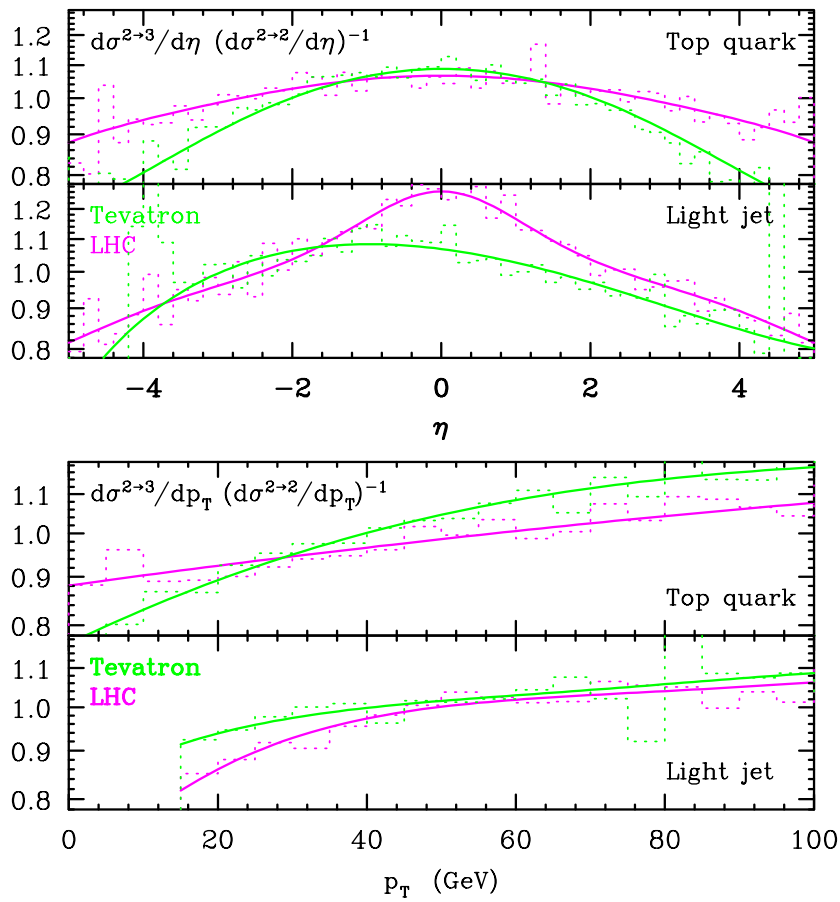

FIG. 4 (color online). Shape comparison for the top quark and light jet NLO distributions. The bin-by-bin ratio of the normalized $\left(4 \mathrm{~F}\right.$ and $5 \mathrm{~F}$ ) distributions in $\eta$ and $p_{T}$ is shown. 

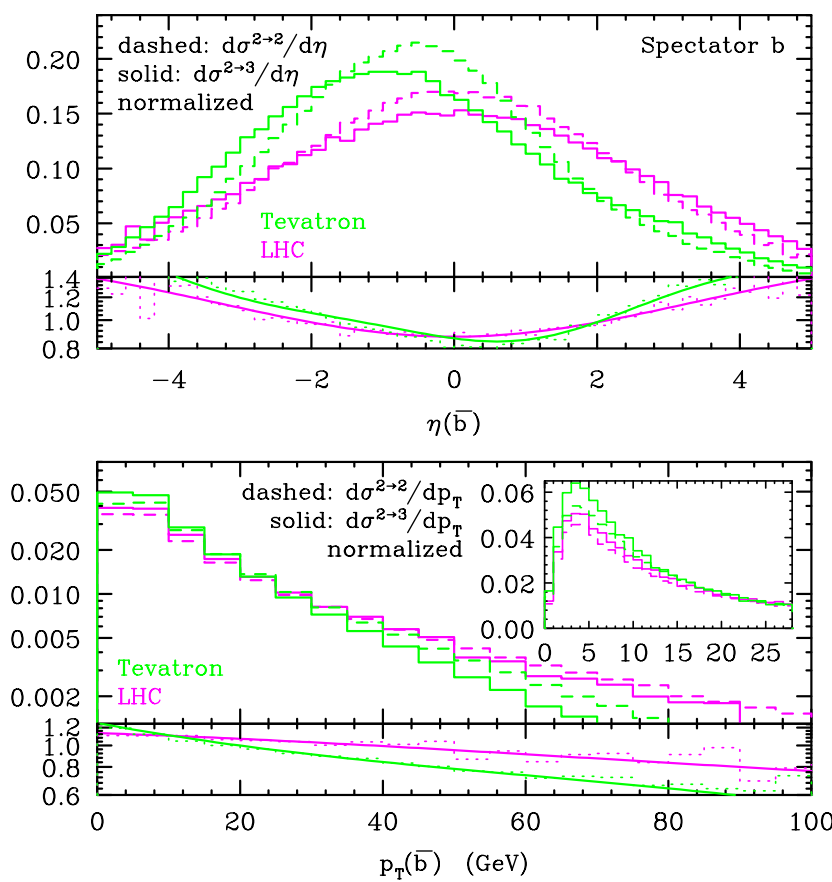

FIG. 5 (color online). Distributions (upper plots) in $\eta$ and $p_{T}$ and shape comparison (lower plots) of the $2 \rightarrow 3$ and $2 \rightarrow 2$ calculations of the spectator $b$ at NLO.

We have reported on the computation of the NLO corrections to the EW production of top and bottom quarks through the $t$-channel exchange of a $W$ boson, keeping the mass of the heavy quarks finite. This allows a systematic study of the approximations and improvements associated with the different schemes for treating heavy flavors in QCD. We find that the $4 \mathrm{~F}$ calculation is well behaved: it displays a $10 \%(4 \%)$ scale uncertainty and a modest (very small) increase of the cross section from LO to NLO at the Tevatron (LHC). It gives rates that are slightly smaller than the $5 \mathrm{~F}$ predictions (by about $6 \%$ ). The two calculations are consistent at the Tevatron, where the uncertainty of the $4 \mathrm{~F}$ calculation is similar to their difference and marginally consistent at the LHC, where the estimated uncertainties are much smaller. Such a difference could be interpreted as resulting from the resummation of the $\log \left(Q^{2} / m_{b}^{2}\right)$ in the $5 \mathrm{~F}$ calculation, or as an indication of the need for even higher order corrections. A NNLO prediction in the $5 \mathrm{~F}$ scheme could help in settling this issue. The $4 \mathrm{~F}$ calculation provides reliable predictions for all the relevant differential distributions, in particular, offering for the first time genuine NLO predictions for the spectator $b$ rapidity and $p_{T}$. A detailed comparison with current Monte Carlo based predictions and an extension of this study to the production of fourth generation quarks, such as $t^{\prime} b, t b^{\prime}$, and $t^{\prime} b^{\prime}$ are left to forthcoming studies.

We are indebted to Scott Willenbrock for numerous comments and insights. We also thank Carlo Oleari and Paolo Nason for their valuable help in comparing with the calculation of Ref. [30]. This work is partially supported by the Belgian Federal Office for Scientific, Technical and
Cultural Affairs through the Interuniversity Attraction Pole No. P6/11.

[1] S. S. D. Willenbrock and D. A. Dicus, Phys. Rev. D 34, 155 (1986).

[2] C. P. Yuan, Phys. Rev. D 41, 42 (1990).

[3] R. K. Ellis and S. J. Parke, Phys. Rev. D 46, 3785 (1992).

[4] V. M. Abazov et al. (D0 Collaboration), Phys. Rev. Lett. 98, 181802 (2007).

[5] T. Aaltonen et al. (CDF Collaboration), Phys. Rev. Lett. 101, 252001 (2008).

[6] J. Alwall et al., Eur. Phys. J. C 49, 791 (2007).

[7] T. Stelzer, Z. Sullivan, and S. Willenbrock, Phys. Rev. D 58, 094021 (1998).

[8] M. T. Bowen, S. D. Ellis, and M. J. Strassler, Phys. Rev. D 72, 074016 (2005).

[9] T. M. P. Tait and C.P. Yuan, Phys. Rev. D 63, 014018 (2000).

[10] G. Bordes and B. van Eijk, Nucl. Phys. B435, 23 (1995).

[11] T. Stelzer, Z. Sullivan, and S. Willenbrock, Phys. Rev. D 56, 5919 (1997).

[12] B. W. Harris, E. Laenen, L. Phaf, Z. Sullivan, and S. Weinzierl, Phys. Rev. D 66, 054024 (2002).

[13] N. Kidonakis, Phys. Rev. D 74, 114012 (2006).

[14] J. M. Campbell, R. K. Ellis, and F. Tramontano, Phys. Rev. D 70, 094012 (2004).

[15] J. M. Campbell and F. Tramontano, Nucl. Phys. B726, 109 (2005).

[16] Q.-H. Cao, R. Schwienhorst, and C. P. Yuan, Phys. Rev. D 71, 054023 (2005).

[17] Q.-H. Cao, R. Schwienhorst, J. A. Benitez, R. Brock, and C. P. Yuan, Phys. Rev. D 72, 094027 (2005).

[18] S. Frixione, E. Laenen, P. Motylinski, and B. R. Webber, J. High Energy Phys. 03 (2006) 092.

[19] S. Frixione, E. Laenen, P. Motylinski, B. R. Webber, and C. D. White, J. High Energy Phys. 07 (2008) 029.

[20] J. C. Collins, Phys. Rev. D 58, 094002 (1998).

[21] M. Kramer, F. I. Olness, and D. E. Soper, Phys. Rev. D 62 , 096007 (2000).

[22] J. A. M. Vermaseren, arXiv:math-ph/0010025.

[23] J. Alwall et al., J. High Energy Phys. 09 (2007) 028.

[24] G. Passarino and M. J. G. Veltman, Nucl. Phys. B160, 151 (1979).

[25] R. K. Ellis and G. Zanderighi, J. High Energy Phys. 02 (2008) 002.

[26] S. Catani and M.H. Seymour, Nucl. Phys. B485, 291 (1997).

[27] S. Catani, S. Dittmaier, M. H. Seymour, and Z. Trocsanyi, Nucl. Phys. B627, 189 (2002).

[28] J. M. Campbell and R. K. Ellis, Phys. Rev. D 62, 114012 (2000).

[29] R. Frederix, T. Gehrmann, and N. Greiner, J. High Energy Phys. 09 (2008) 122.

[30] P. Nason and C. Oleari, Nucl. Phys. B521, 237 (1998).

[31] P. M. Nadolsky et al., Phys. Rev. D 78, 013004 (2008).

[32] M. Cacciari, M. Greco, and P. Nason, J. High Energy Phys. 05 (1998) 007.

[33] A. D. Martin, W. J. Stirling, and R. S. Thorne, Phys. Lett. B 636, 259 (2006).

[34] Z. Sullivan, Phys. Rev. D 70, 114012 (2004). 